\title{
Inertial control as novel technique for in vitro gait simulations
}

\author{
T. Natsakis ${ }^{a, *}$, J. Burg ${ }^{\mathrm{a}, \mathrm{b}}$, G. Dereymaeker ${ }^{\mathrm{a}}$, I. Jonkers ${ }^{\mathrm{b}}$, J. Vander Sloten ${ }^{\mathrm{a}}$ \\ ${ }^{a}$ KU Leuven, Department of Mechanical Engineering, Celestijnenlaan 300c, Box 2419, 2001 Heverlee, Belgium \\ ${ }^{b}$ KU Leuven, Faculty of Kinesiology and Rehabilitation Science, Tervuursevest 101, Box 1500, 3001 Heverlee, Belgium
}

\begin{abstract}
In vitro gait simulations are a preferential platform to study new intervention techniques or surgical procedures as they allow studying the isolated effect of surgical interventions. Commonly, simulations are performed by applying pre-defined setpoints for the kinetics and kinematics on all degrees of freedom (DOFs) of the cadaveric specimen. This however limits the applicability of the experiment to simulations for which pre-defined kinematics and kinetics can be measured in vivo. In this study we introduce inertial control as a new methodology for gait simulations that omits the need for pre-defined setpoints for the externally applied vertical ground reaction force (vGRF) and therefore allows the effect of interventions to be reflected upon it. Gait simulations of stance $(1 \mathrm{sec})$ were performed in 10 cadaveric specimens under three clinically relevant conditions: native ankle, total ankle prosthesis (TAP) and total ankle prosthesis plus triple arthrodesis (TAP+TA) (TAP+TA). In the native ankle, simulated vGRF were compared against the vGRF measured in vivo in 15 healthy volunteers and high correlations were found $\left(R^{2}=0.956\right.$, slope of regression line $\mathrm{S}=1.004$ ). In TAP and TAP+TA, vGRF changed, therefore confirming the sensitivity of the method to kinematic constrains imposed with surgery. Inertial control can replicate in vivo kinetic conditions and allows investigating the isolated effect of surgical interventions on kinematic as well as kinetics.
\end{abstract}

Keywords: Gait simulations, in vitro, control theory, total ankle prosthesis, triple arthrodesis

\section{Introduction}

When studying surgical interventions on the foot, in vitro gait simulations allow isolating the effect of surgery on the resulting kinematics and kinetics $[2,12,14,16]$. This is highly relevant as it is known from literature $[13,18,15]$ that factors such as time since operation, pain, muscle training etc., can influence the measured kinematics and kinetics in patients. Therefore, the isolated effect of a surgical intervention cannot be studied in vivo.

During current in vitro gait simulations, input set points derived from a control group are applied in all degrees of freedom (DOFs) of the specimen. These set-points are in the form of either tibial kinematics $[1,7,11]$ or a combination of tibial kinematics and vertical ground reaction force (vGRF) [3, 6]. This, however, limits the applicability to simulations where it is of interest to impose pre-defined kinematics and kinetics measured in vivo, e.g. when studying bone kinematics during normal gait. When however, the sole effect of an intervention on the kinematics or kinetics is studied, this approach cannot be used. The simulation is over-constrained and it does not allow the effect of the specific intervention to be reflected in the kinematics and kinetics as they are imposed and not measured.

To overcome this limitation, we present a new technique that alleviates the need for a predefined set-point for the vertical tibial kinematics or vGRF during in vitro gait simulations, which leaves one flexible DOF for the effect of the interventions to be reflected upon. To demonstrate the applicability of the technique, gait simulations were performed in intact cadaveric foot specimens and the resulting vGRF was evaluated. To motivate its clinical relevance, vGRF were also evaluated during gait simulations with identical input

*Corresponding author 


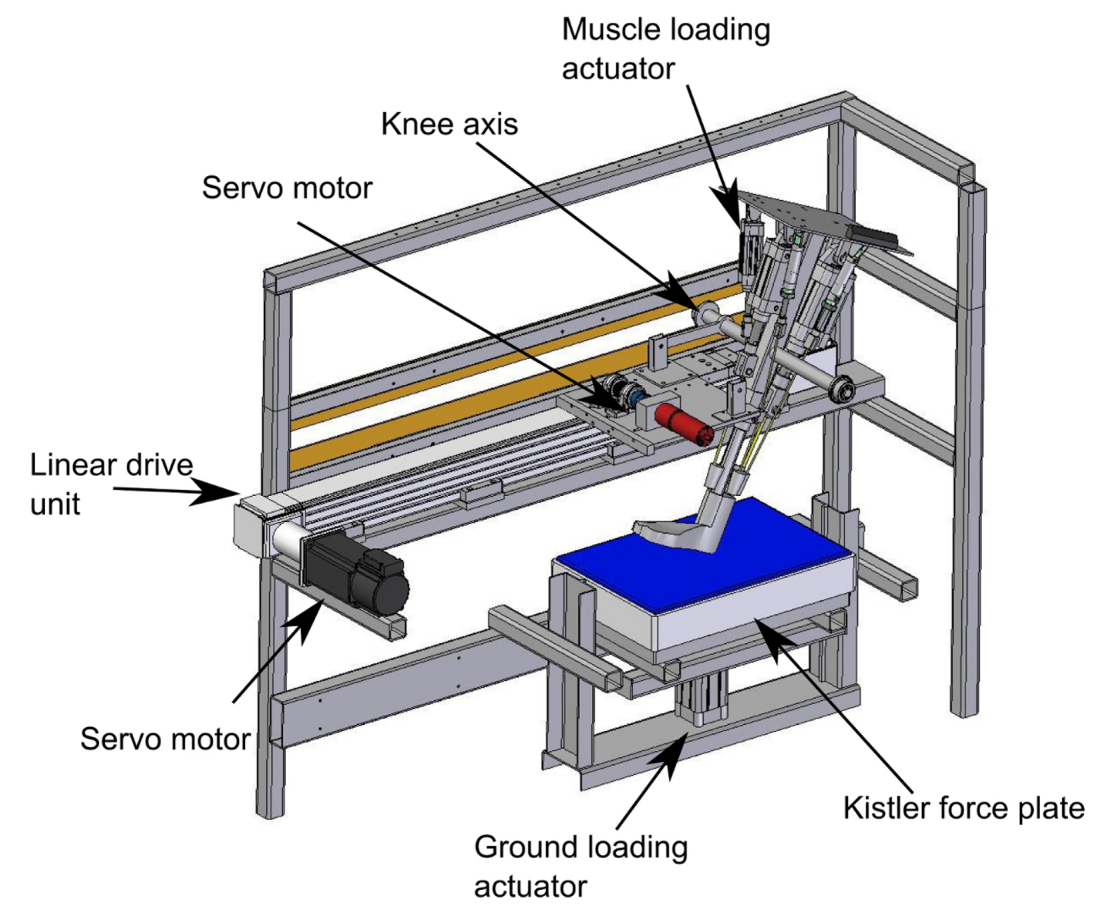

Figure 1: The CGS. In the figure, the motor imposing the horizontal motion (black), the sliding rail in the horizontal direction, the carriage with the actuators, the tibial rotation motor (red) and the supporting plate (blue) of the foot are presented

parameters but after applying a total ankle prosthesis (TAP) and total ankle prosthesis plus triple arthrodesis $(\mathrm{TAP}+\mathrm{TA})(\mathrm{TAP}+\mathrm{TA})$ locking the hindfoot motion of the specimen.

\section{Methods}

The inertial control approach is applied on a custom built cadaveric gait simulator (CGS) that manipulates the sagittal plane tibial kinematics of cadaveric foot specimens (figure 1). Even though the remaining 3 DOF are constrained, previous validation studies $[8,5]$ demonstrated that the CGS is able to reconstruct kinematics similar to those measured in vivo. To account for the effect of plantarflexion and the resulting up and downwards translation of the knee axis, a supporting plate parallel to the ground translates vertically and modifies the foot position. This motion is imposed by a pneumatic actuator (Festo ADNGF-63-100-P-A, Sankt Ingbert, Germany) operating in a force feedback loop. The force applied by the actuator is determined by the inertial controller. A force plate (9281 B Kistler Multicomponent Force Plate, Kistler Instruments $\mathrm{GmbH}$, Germany), mounted on top of the supporting platform, contacts the foot and measures the vGRF at $2 \mathrm{kHz}$. Stance phase is simulated in one second and the horizontal translation and sagittal plane rotation of the tibia as well as the activation of 6 muscle groups (peroneal muscles, tibialis anterior, tibialis posterior, flexor digitorum, triceps surae and flexor halucis) are controlled based on prescribed set-points. The vertical kinematics of the tibia imposed by the translating ground plate is defined using the inertial controller approach. All devices are operated and synchronised by a custom built controller programmed in LabVIEW 2013 (National Instruments, TX, USA).

In vivo, the vGRF exerted on each foot during walking is expressed as in equation (1) [10] (figure 2a), with $m_{h}$ the mass of the person, $a_{h}(x)$ the vertical acceleration of the bodys centre of gravity, W(x) the portion of the weight of the person supported by that foot and $\mathrm{x}$ the percentage of stance phase.

$$
v \operatorname{RRF}_{\text {invivo }}(x)=W(x)+m_{h} * a_{h}(x)
$$




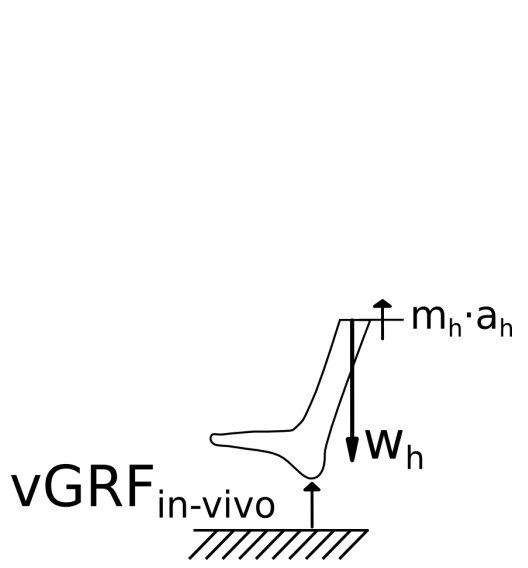

(a)

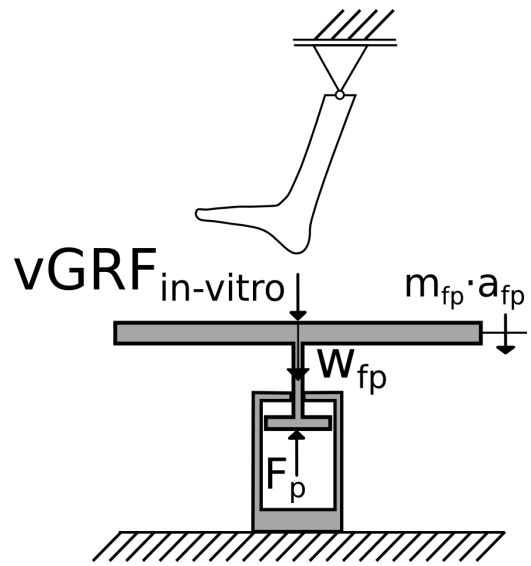

(b)

Figure 2: Free body diagram of (a) the foot and (b) the supporting plate of the CGS. The force of the actuator $\left(F_{p}\right)$, the weight of the plate $\left(W_{f p}\right)$ and the reaction force of the foot $\left(\mathrm{vGRF}_{\text {invitro }}\right)$ impose an acceleration $\left(a_{f p}\right)$ on the mass of the plate $\left(m_{f p}\right)$. The force delivered by the plate to the foot is equal in magnitude and opposite in direction to the force delivered by the foot to the plate $\left(\mathrm{vGRF}_{\text {invitro }}\right)$. The accelerations have different directions between in vitro and in vivo cases, as downward movement of the plate is equivalent to an upward movement of the knee.

$W(x)$ is a function of the percentage of stance phase $(x)$, with weight increasing from 0 to $100 \%$ body weight (BW) during the first $16 \%$ of the stance phase (initial double support (IDS)) and decreasing back to $0 \% \mathrm{BW}$, during the final $16 \%$ (terminal double support (TDS)) [10]. The duration of the IDS and TDS was chosen for normal gait conditions, it can however be adapted to correspond to the value observed in a specific patient group. Assuming continuity and smoothness during this transition, the weight carried by the foot during stance phase is described by equation (2) where $f(x)$ is an algebraic sigmoid function with input $\mathrm{x}$ being the stance phase percentage (equation (3)) (figure 3).

$$
\begin{array}{r}
v G R F_{\text {invivo }}(x)=W_{h} * f(x)+m_{h} * a_{h}(x) \\
f(n)=\left\{\begin{array}{cl}
\frac{0.5 x^{2}}{x^{2}-184 x+128}, & \text { if } x<16 \\
1, & \text { if } 16 \leq x \leq 84 \\
\frac{0.5(x-100)^{2}}{x^{2}-184 x+8528}, & \text { if } x>84
\end{array}\right.
\end{array}
$$

Based on the free body diagram of the supporting plate of the CGS (figure 2b), equation (4) can be composed. The force $\left(F_{p}\right)$ applied by the actuator on the plate is controlled to be equal to the weight of the plate $\left(W_{f p}\right)$, plus a simulated weight of the specimen $\left(W_{s w}\right)$ multiplied by the sigmoid function $(f(x))$ to simulate vGRF during double support (equation (5)). To ensure cadaveric integrity, the simulation weight was reduced to $50 \%$ of the control subject weight $(49.7 \mathrm{~kg})$, and a similar reduction was applied to the muscle forces $\left(W_{s w}=240 N\right)$. Combining equation (4) and equation (5) into equation (6), the vGRF profile corresponding to the in vivo situation (equation (2)) is simulated.

$$
\begin{array}{r}
v G R F_{\text {invitro }}(x)-F_{p}(x)+W_{f p}=m_{f p} * a_{f p}(x) \\
F_{p}(x)=W_{f p}+W_{s w} * f(x) \\
v G R F_{\text {invitro }}(x)=W_{s w} * f(x)+m_{f p} * a f p(x)
\end{array}
$$

Following equation (6) the mass $\left(m_{f p}\right)$ and acceleration $\left(a_{f p}\right)$ of the force plate corresponds to the mass $\left(m_{h}\right)$ and acceleration $\left(a_{h}\right)$ of the body's centre of mass during normal walking. More specific, to mimic the 


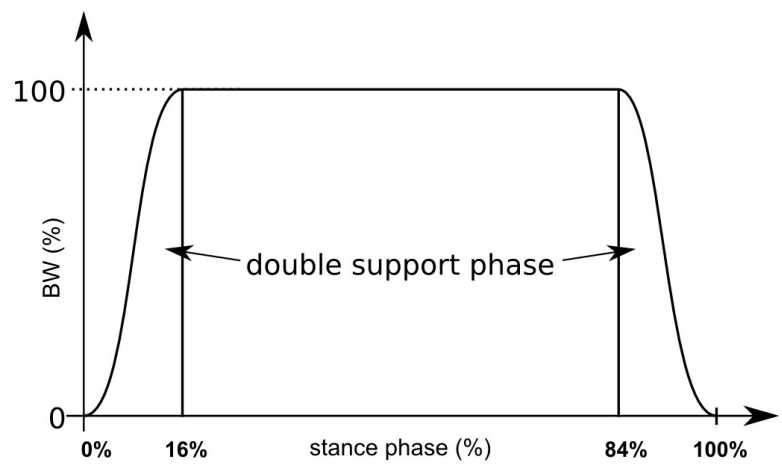

Figure 3: The function $f(x)$ of the weight carried by the foot during stance phase. The sigmoid parts are visible during the transition period of the double supported phases. The function is continuous and smooth over the whole duration of stance phase.

upwards acceleration of the centre of mass during push off, the force plate will move down with the inertia of the supporting plate contributing to the vGRF during simulated gait.

To evaluate the proposed methodology and to demonstrate a clinically relevant example, in vitro gait simulations were performed in 10 freshly frozen cadaveric foot specimens using the inertial control approach under three different conditions. The feet were initially tested intact and afterwards, a three component TAP (Hintegra, New Deal, Lyon, France) was implanted, followed by a triple arthrodesis between the bones of the hind foot (TAP+TA). All surgical procedures were performed by an experienced foot surgeon and 15 repetitions were performed for each condition. All repetitions were used during the analysis of the results.

The results were compared against vGRF that was captured in vivo in 15 healthy subjects, using two force plates (AMTI OR6 series, AMTI, MA, USA) at $1 \mathrm{kHz}$ and normalised for BW. Since the weight of the person $\left(W_{h}\right)$ is equal to the mass $\left(m_{h}\right)$ multiplied by the gravitational acceleration $\left(g=9.8 \mathrm{~m} / \mathrm{s}^{2}\right)$ (equation (7)), the normalised forces, based on equation (2), are of the form (equation (8)).

$$
\begin{array}{r}
W_{h}=m_{h} * g \\
G_{\text {norm }, \text { invivo }}(x)=\frac{W_{h} * f(x)+m_{h} * a_{h}(x)}{W_{h}}=f(x)+\frac{a_{h}(x)}{g}
\end{array}
$$

For the in vitro measurements, a difference between the simulated weight $\left(W_{s w}=240 N\right)$ and the mass of the platform $\left(m_{f p}=70 \mathrm{~kg}\right)$ may arise. Therefore, a different normalisation approach was used. The simulated weight over stance phase function $\left(W_{s w} * f(x)\right)$ was first subtracted from the measured vGRF and the result was divided by the mass of the force plate $\left(m_{f p}\right)$ multiplied by the gravitational force $(\mathrm{g})$. Finally, the sigmoid function $\mathrm{f}(\mathrm{x})$ was added, so that it corresponded with the normalised in vivo measurements (equation (9)).

$$
\begin{array}{r}
v G R F_{\text {norm }, \text { invitro }}(x)= \\
\frac{v G R F_{\text {dynamic,invitro }}-W_{s w} * f(x)}{m_{f p} * g}+f(x)=f(x)+\frac{a_{f p}(x)}{g}
\end{array}
$$

Using a non-parametric rank sum test (Wilcoxon) for each percentage of stance phase, the intact in vitro vGRF was compared against intact in vivo condition and the TAP in vitro and TAP + TA in vitro conditions were compared against intact in vitro. Statistically significant differences were reported for $p<0.05$. A linear regression was finally performed within the mean values of intact in vivo and in vitro measurements. The statistical analysis was performed with $\mathrm{R}$ v3.0.2 [9]. 


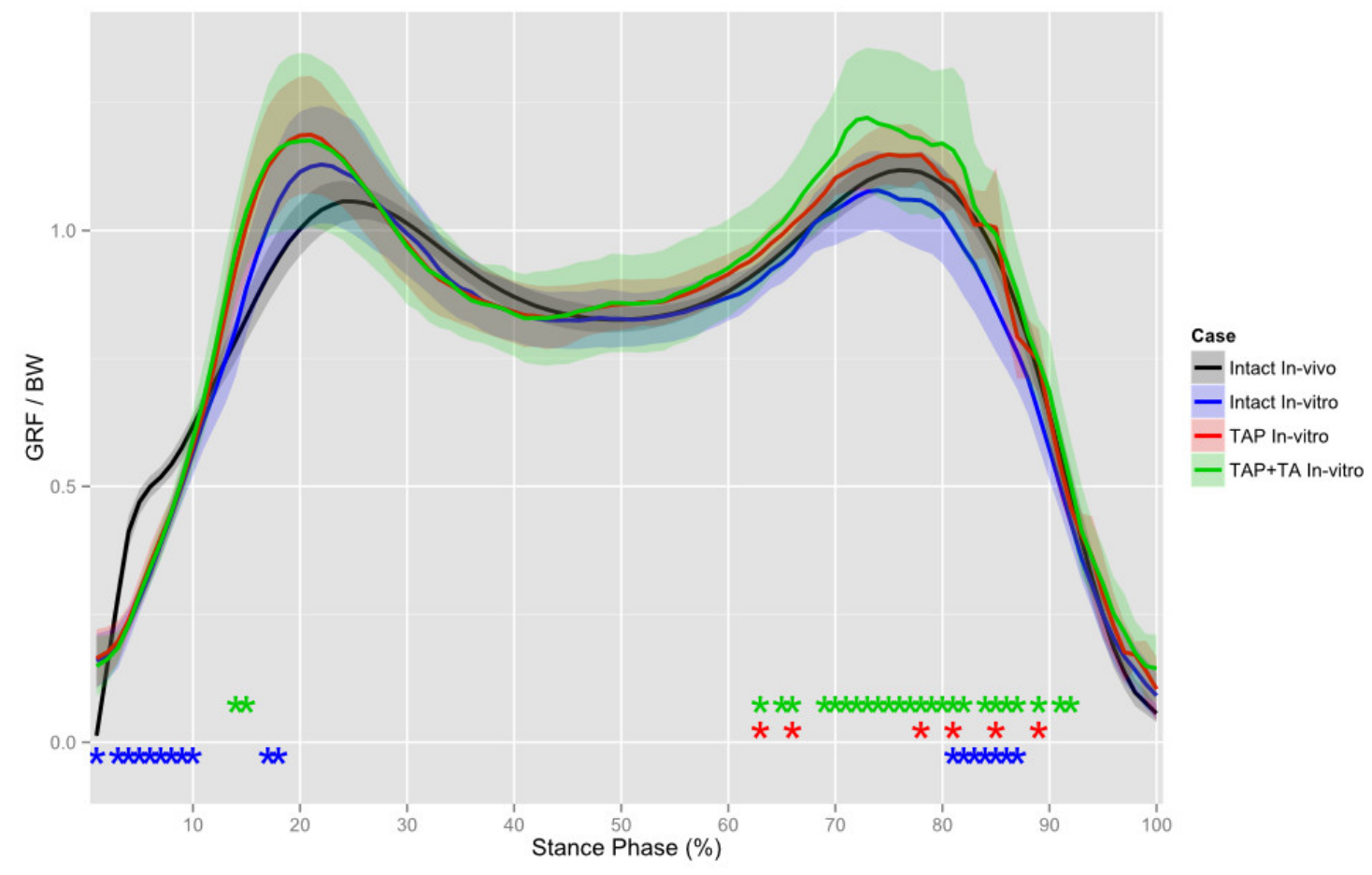

Figure 4: Normalised vGRF of intact in vivo (black), intact in vitro (blue), TAP in vitro (red) and TAP+TA in vitro (green) groups. The average vGRF and the standard deviation of all feet and repetitions are presented. Significant differences between the intact in vivo and in vitro (blue), the intact in vitro and TAP in vitro (red) and the intact in vitro and TAP+TA in vitro (green) measurements are marked with an asterix $(*)$.

\section{Results}

The normalised signals of the vGRF obtained from the in vitro simulations and in vivo gait analysis, are presented in figure 4 . Timing of the significant differences is indicated using an asterix $(*)$. For the intact ankle, significant differences in $\mathrm{vGRF}$ of the intact in vivo and intact in vitro data are primarily found during the first $10 \%$ and between $80 \%$ and $90 \%$ of stance phase. Only isolated differences from $62 \%$ to $90 \%$ are found between the intact in vitro and TAP in vitro measurements. More significant differences are found comparing the intact in vitro and TAP+TA in vitro measurements around the first $(13 \%-14 \%)$ and especially around the second peak (63\%-92\%). The regression between the intact in vitro and intact in vivo was $R^{2}=0.956$ with slope $\mathrm{S}=1.004$.

\section{Discussion}

Reproducing physiologic gait in cadaveric specimens poses many challenges and several research groups have developed custom built cadaveric gait simulators trying to address them $[17,7,6,11,3,4]$. In several designs, physiologic tibial kinematics must be applied, coupled with adequate force production to the different tendon actuators. These input variables must be synchronised and applied accurately. Furthermore, these signals must be appropriate for each cadaveric specimen, to account for differences in the geometry and condition [5]. Finally, when the interest of a study is to investigate the isolated effect of a parameter (e.g. surgical intervention) the simulations should not be over-constrained to allow the phenomena that are studied to be reflected on the signals that are being measured.

In this study, we evaluate a methodology for performing gait simulations on cadaveric specimens without the need of applying predefined vGRF. We evaluate its sensitivity in two specific interventions and to isolate 
their effect, muscle forces remain unaltered across conditions. Gait was simulated in intact ankle, TAP and TAP+TA joint, allowing one DOF to adapt (vGRF and vertical kinematics) and controlling the remaining DOF (horizontal kinematics, tibial rotation) and muscle actuation with identical setpoints across different conditions. We, therefore, assumed that the changes in the biomechanics of the foot introduced by the subsequent interventions would be reflected upon the measured vGRF, as this illustrates the sensitivity of this methodology.

The results from this methodology were compared against data captured during in vivo gait analysis. The high $R^{2}$ values $\left(R^{2}=0.956\right)$ observed between the intact in vivo and intact in vitro group, indicates that the proposed methodology accurately reproduces physiological vGRF. The vGRF between the two groups was significantly different during limited part of stance phase i.e. just before the 1st and slightly after the 2nd peak. This however does not limit the validity of the methodology, as the magnitude of the differences is low and they appear during very short stance phase intervals. Furthermore, the changes in magnitude of the vGRF following the subsequent surgical procedures, demonstrates the sensitivity of the methodology to changes in the condition of the foot. The less pronounced differences between the intact in vitro and TAP in vitro than between intact in vitro and TAP + TA in vitro groups are introduced as the triple arthrodesis, that locks different midfoot joints, constrains the foot motion more with larger impact on the kinematics compared to TAP.

To the best of our knowledge, this study is the first presenting such a methodology, which opens new possibilities in in vitro gait simulations e.g studying the effect of new implant design, muscle activation or surgical interventions when the effect of the intervention needs to be isolated and not affected by pain or fear of a patient. vGRF obtained from our CGS using the inertial controller show equally high correspondence with vGRF obtained from in vivo gait analysis with those reported in other studies. Furthermore, the simulation speed is increased compared to previous designs, being very close to physiologic speed. Finally, with the need for a pre-defined set-point removed for the vGRF, the inertial controller provides opportunities to simulate gait under different conditions and investigate surgical interventions or new implant designs and their influence on gait parameters.

\section{Acknowledgements}

This work was funded by the Chair Berghmans- Dereymaeker, the Research Foundation Flanders and the Agency for Innovation by Science and Technology in Flanders (IWT). The authors would like to thank Pieter Spaepen (KU Leuven, Campus Groep T, Belgium) for his valuable feedback during the development of this methodology.

\section{Conflict of interest}

The authors have no conflicts of interest to report.

\section{Bibliography}

[1] Patrick M. Aubin, Eric Whittaker, and William R. Ledoux. A Robotic Cadaveric Gait Simulator With Fuzzy Logic Vertical Ground Reaction Force Control. IEEE Trans. Robot., 28(1):246-255, February 2012. ISSN 1552-3098. doi: 10.1109/TRO.2011.2164958.

[2] Ahmad F. Bayomy, Patrick M. Aubin, Bruce J. Sangeorzan, and William R. Ledoux. Arthrodesis of the First Metatarsophalangeal Joint: A Robotic Cadaver Study of the Dorsiflexion Angle. J. Bone Jt. Surg., 92(8):1754-1764, 2010. doi: 10.2106/JBJS.I.00984.

[3] Christof Hurschler, Judith Emmerich, and Nikolaus Wülker. In vitro simulation of stance phase gait part I: Model verification. Foot Ankle Int., 24(8):614-22, August 2003. ISSN 1071-1007. doi: 10.1177/107110070302400808.

[4] Kyu-Jung Kim, Harold B. Kitaoka, Zong-Ping Luo, Satoru Ozeki, Lawrence J. Berglund, Kenton R. Kaufman, and An Kai-Nan. In vitro simulation of the stance phase in human gait. J. Musculoskelet. Res., 5(2):113-121, 2001. doi: 10.1142/S0218957701000490.

[5] Tassos Natsakis, Koen Peeters, Fien Burg, G. Dereymaeker, Jos Vander Sloten, and Ilse Jonkers. Specimen-specific tibial kinematics model for in vitro gait simulations. Proc. Inst. Mech. Eng. Part H J. Eng. Med., 227(4):454-463, November 2012. ISSN 0954-4119. doi: 10.1177/0954411912464459. 
[6] Chris J. Nester, Richard K. Jones, A.M. Liu, David Howard, A. Lundberg, A. Arndt, P. Lundgren, A. Stacoff, and P. Wolf. Foot kinematics during walking measured using bone and surface mounted markers. J. Biomech., 40(15):3412-3423, 2007. ISSN 0021-9290. doi: doi:DOI:10.1016/j.jbiomech.2007.05.019.

[7] Lawrence D. Noble, Robb W. Colbrunn, Dong-Gil Lee, Antonie J. van den Bogert, and Brian L. Davis. Design and validation of a general purpose robotic testing system for musculoskeletal applications. J. Biomech. Eng., 132(2):02500112, February 2010. ISSN 1528-8951. doi: 10.1115/1.4000851.

[8] Koen Peeters, Tassos Natsakis, Josefien Burg, Pieter Spaepen, Ilse Jonkers, Greta Dereymaeker, and Jos Vander Sloten. An in vitro approach to the evaluation of foot-ankle kinematics: performance evaluation of a custom-built gait simulator. Proc. Inst. Mech. Eng. Part H J. Eng. Med., 227(9):955-67, September 2013. ISSN 2041-3033. doi: 10.1177/0954411913490455.

[9] R Core Team. R : A Language and Environment for Statistical Computing, 2013.

[10] Jim Richards. Biomechanics in Clinic and Research: An interactive teaching and learning course. Churchill Livingstone/Elsevier, 2008, London, first edition, 2008. ISBN 978-0-443-10170-0.

[11] Neil A. Sharkey and Andrew J. Hamel. A dynamic cadaver model of the stance phase of gait: performance characteristics and kinetic validation. Clin. Biomech., 13(6):420-433, September 1998. ISSN 0268-0033. doi: 10.1016/S0268-0033(98) 00003-5.

[12] Andreas Suckel, Otto Muller, Tina Herberts, Peter Langenstein, Patrik Reize, and Nikolaus Wulker. Talonavicular arthrodesis or triple arthrodesis: peak pressure in the adjacent joints measured in 8 cadaver specimens. Acta Orthop., 78 (5):592-7, October 2007. ISSN 1745-3674. doi: 10.1080/17453670710014275.

[13] Rhys Thomas, Tim R Daniels, and Kim Parker. Gait analysis and functional outcomes following ankle arthrodesis for isolated ankle arthritis. J. Bone Joint Surg. Am., 88(3):526-35, March 2006. ISSN 0021-9355. doi: 10.2106/JBJS.E.00521.

[14] Victor Valderrabano, Beat Hintermann, Benno M Nigg, Darren Stefanyshyn, and Pro Stergiou. Kinematic changes after fusion and total replacement of the ankle: part 1: Range of motion. Foot Ankle Int., 24(12):881-7, December 2003. ISSN 1071-1007.

[15] Victor Valderrabano, Benno M Nigg, Vinzenz von Tscharner, Darren J Stefanyshyn, Beat Goepfert, and Beat Hintermann. Gait analysis in ankle osteoarthritis and total ankle replacement. Clin. Biomech. (Bristol, Avon), 22(8):894-904, October 2007. ISSN 0268-0033. doi: 10.1016/j.clinbiomech.2007.05.003.

[16] Joel R Weber, Patrick M Aubin, William R Ledoux, and Bruce J Sangeorzan. Second metatarsal length is positively correlated with increased pressure and medial deviation of the second toe in a robotic cadaveric simulation of gait. Foot Ankle Int., 33(4):312-9, April 2012. ISSN 1071-1007. doi: 10.3113/FAI.2012.0312.

[17] Eric C. Whittaker, Patrick M. Aubin, and William R. Ledoux. Foot bone kinematics as measured in a cadaveric robotic gait simulator. Gait Posture, 33(4):645-650, April 2011. ISSN 0966-6362. doi: 10.1016/j.gaitpost.2011.02.011.

[18] W L Wu, F C Su, Y M Cheng, P J Huang, Y L Chou, and C K Chou. Gait analysis after ankle arthrodesis. Gait Posture, 11(1):54-61, February 2000. ISSN 0966-6362. 